\title{
Comparison of access window created by cervical ventral slot and modified slanted ventral slot in canine cadaver
}

\author{
Ho Young Kang', Jinsu Kang', Haebeom Lee ${ }^{2}$, Namsoo Kim¹, Suyoung Heo ${ }^{1 *}$ \\ ${ }^{1}$ College of Veterinary Medicine, Jeonbuk National University, Iksan 54596, Korea \\ ${ }^{2}$ College of Veterinary Medicine, Chungnam National University, Dajeon 34134, Korea
}

\begin{abstract}
Ten cadavers were studied to compare the accessibility of cervical vertebral canal with conventional standard ventral slot and modified slanted ventral slot. One group performed standard ventral slot procedures at the C3-4, C5-6. The other group performed modified slanted ventral slot with inverted cone technique procedures at the C3-4, C5-6 computed tomography was performed before and after surgery. The accessibility of cervical vertebral canal with conventional standard ventral slot and modified slanted ventral slot using computed tomography in C3-4 and C5-6 intervertebral space was compared. Although smaller ostectomy was performed in the modified slanted ventral slot, some lesions were more accessible but limitations were obvious in C3-4 and C5-6 intervertebral disk space. After the disc material has been identified through accurate preoperative diagnostic imaging, less morbidity and complications can be expected if the appropriate surgical method is selected based on the lesion of compression.
\end{abstract}

Keywords: cervical disc herniation, ventral slot, modified slanted ventral slot, accessibility, dog

*Corresponding author

Suyoung Heo

College of Veterinary Medicine, Jeonbuk National University, Iksan 54596, Korea

Tel: $+82-63-850-0954$

Fax: +82-63-850-0972

E-mail: syheo@jbnu.ac.kr

ORCID:

Ho Young Kang

https://orcid.org/0000-0001-6076-3673

Jinsu Kang

https://orcid.org/0000-0001-5501-7983

Haebeom Lee

https://orcid.org/0000-0002-1594-6670

Namsoo Kim

https://orcid.org/0000-0003-2160-1203

Suyoung Heo

https://orcid.org/0000-0002-7733-3263

Conflict of Interest

The authors declare no conflicts of interest.

Received: June 27, 2019

Revised: June 15, 2020

Accepted: July 20, 2020

\section{Introduction}

Cervical intervertebral disc herniation is a common disease condition in dogs [1]. Some $14 \%$ to $25 \%$ of dogs with intervertebral disc herniation have cervical intervertebral disc herniation [2]. The most common disc disease in small breed dogs is Hansen type 1 (extrusion), which commonly affects the C3-4 intervertebral disc space. In large breed dogs, cervical disc disease usually occurs at the C5-6 intervertebral disc space and the type of disc herniation is commonly Hansen type 2 (protrusion) [3].

When cervical intervertebral disc disease (IVDD) occurs, conservative treatment or surgical treatment can be performed depending on the condition of the patient $[1,4]$. Conservative procedures include restriction/cage rest, and use of anti-inflammatory medications such as corticosteroids or nonsteroidal anti-inflammatory drugs $[2,4]$. Based on multiple factors, the methods that can be performed when surgery is necessary are ventral slot, slanted ventral slot, dorsal laminectomy, hemilaminectomy, and fenestration [5]. Among the various methods, the ventral slot technique is the preferred method for decompression of most cervical spinal cord lesions that result from intervertebral disease [5].

There are many complications reported following use of the ventral slot technique, including hemorrhage, Honer's syndrome, hypotension, vertebral subluxation and instability [6-8]. To overcome these complications, alternative techniques, including slanted ventral slot, modified slanted ventral slot and inverted cone decompression are suggested. These techniques have been proposed to reduce the complications associated with standard ventral slots, performing effective decompression through less invasive ostectomy $[9,10]$. Modified slanted ventral slot is a method that combines the slanted ventral slot and the inverted cone decompression techniques. In 2007, one study reported that modified slanted ventral slot reduced complications compared to standard ventral slot [9]. However, there is no study comparing the standard ventral slot and modified slanted ventral slot techniques in terms of accessibility of the cervical vertebral canal. 
The purpose of this study is to compare using computed tomography (CT) the results of performing either the standard ventral slot or the modified slanted ventral slot techniques in the C3-4 and C5-6 intervertebral spaces in terms of the accessibility of the cervical vertebral canal. The C3-4 and C5-6 intervertebral spaces were chosen because they are a common site for disc associated disease in small breed dogs and large breed dogs [4].

\section{Materials and Methods}

\section{Cadaver preparations}

Ten canine beagle cadavers that had been euthanized for reasons not related to this study were used. The cadavers, weighing 9.8 to $13.6 \mathrm{~kg}$, were divided into two groups. On one group, the standard ventral slot procedure was performed at the C3-4 and C5-6 intervertebral spaces. On the other group, the modified slanted ventral slot with inverted cone technique was performed at the C3-4 and C5-6 intervertebral spaces.

\section{Surgical procedures}

All standard ventral slot and modified slanted ventral slot with inverted cone procedures were carried out by a single surgeon (HY Kang). Both procedures were performed with a high-speed pneumatic drill with $2 \mathrm{~mm}$ or $4 \mathrm{~mm}$ burr tips and $1 \mathrm{~mm}$ or $2 \mathrm{~mm}$ Kerrison rongeurs. An assistant irrigated the surgical site during drilling using normal saline solution.

\section{Standard ventral slot}

The procedure was performed through a ventral approach to the spine with the cadaver in dorsal recumbency. Before the procedure, a $\mathrm{C}$-arm was used to confirm the surgery site, which was marked on the skin using a surgi-pen. The ventral midline incision was extended from $\mathrm{C} 3$ to C6. After skin incision, the sternohyoid muscles were divided in the midline. Once the sternohyoid muscles had been divided, the trachea and esophagus were retracted. To gain access to the disc, the longus colli muscles were divided in the midline from $\mathrm{C} 3$ to $\mathrm{C} 6$. To perform an accurate procedure, a C-arm was used to re-confirm the surgery site.

The disc was fenestrated using a \#11 blade and mosquito hemostat forceps. After fenestration of the disc, a window was created in the cranial and caudal endplates of the adjacent vertebrae using the high-speed pneumatic drill and burr. The width of the standard ventral slot was maintained at almost $33 \%$ of the width of the body of $\mathrm{C} 3,4,5$, and 6 . Also, the length of the standard slot was maintained at below $33 \%$ of the body of $\mathrm{C} 3,4,5$, and 6 (Fig. 1 A-C). The cancellous bone was removed over the slot area and then thinned dorsal cortical bone was removed using $1 \mathrm{~mm}$ or $2 \mathrm{~mm}$ Kerrison rongeurs.

\section{Modified slanted ventral slot}

Procedures were performed as previously reported for the modified slanted ventral slot technique $[9,10]$ and the inverted cone technique [11]. As with the standard ventral slot procedures, a C-arm was used to confirm the surgery site, which was marked on the skin using a surgi-pen before the procedure. Following a standard ventral slot approach to the cervical vertebrae from $\mathrm{C} 3$ to $\mathrm{C} 6$, the longus muscle was elevated by periosteal elevator. After approach to the cervical vertebrae, the discs of C3-4 and C5-6 were fenestrated using a \#11 blade and mosquito hemostat forceps. By performing fenestration of the disc, it was possible to probe between the disc and monitor the direction and depth of the ostectomy. A window was created a few millimeters away from the cranial vertebral body (C3, C5). The ostectomy window extended
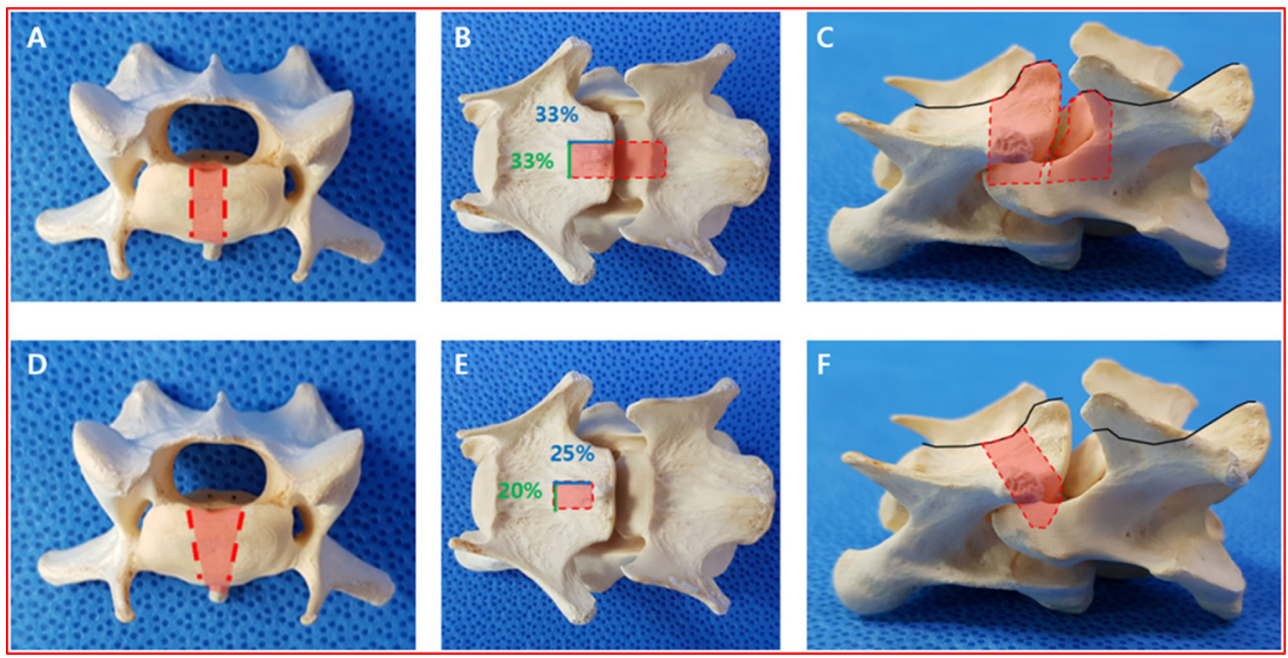

Fig. 1. (A-C), Standard ventral slot technique. (A) Transverse view of C3, (B) ventral view of C 3-4, (C) lateral view of C3-4. The dashed line is the part that ostectomy will be performed. (D-F) Modified slanted ventral slot technique. (D) Transverse view of C3, (E) ventral view of C3-4, (F) lateral view of C3-4. The dashed line is the part that ostectomy will be performed. C, cervical vertebra. 

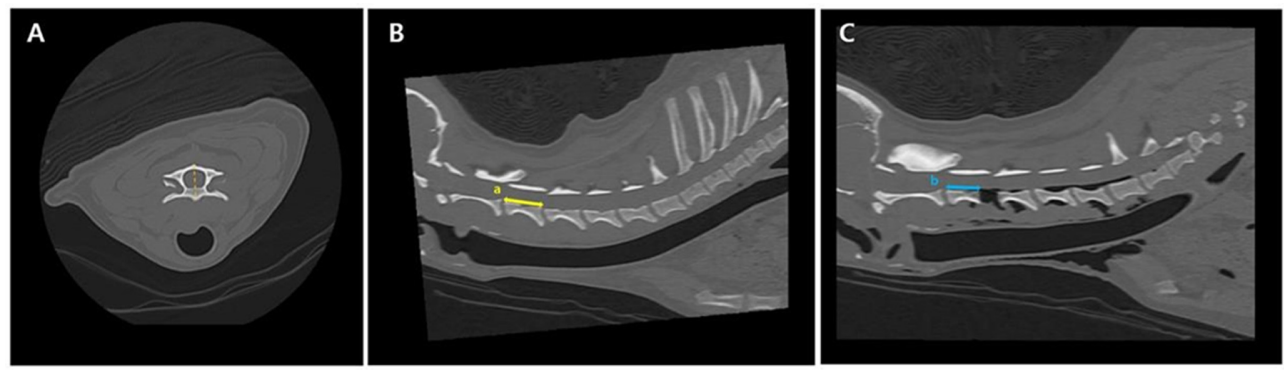

Fig. 2. Standard ventral slot, measurements taken from sagittal slice in C3, 4. (A) Median point of vertebral body, (B, a) intact vertebral body length, $(\mathrm{C}, \mathrm{b})$ sagittal remnant. $\mathrm{C}$, cervical vertebra.
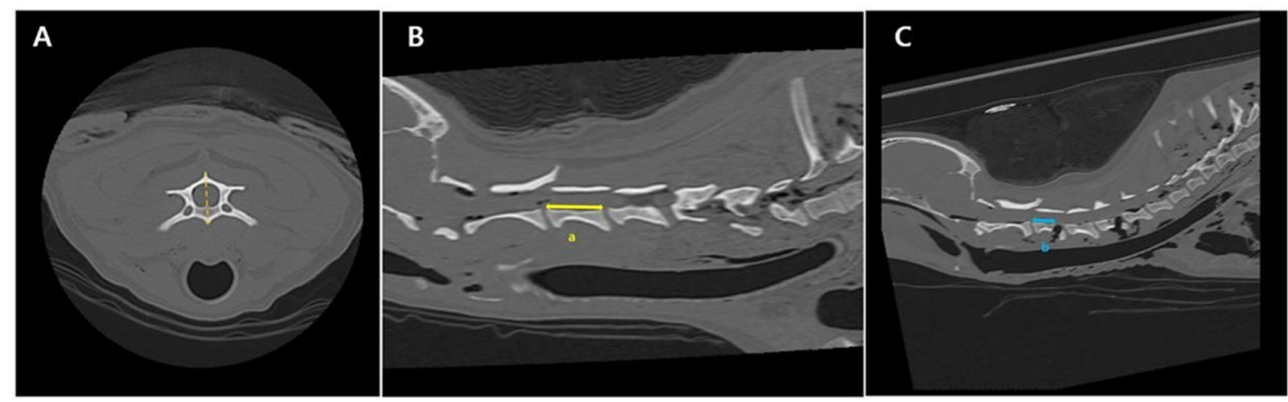

Fig. 3. Modified slanted ventral slot, measurements taken from sagittal slice in C3, 4. (A) Median point of vertebral body, (B, a) intact vertebral body length, $(\mathrm{C}, \mathrm{b})$ sagittal remnant. $\mathrm{C}$, cervical vertebra.

approximately 20 percent of the width and 20-25 percent of the length of the vertebra body (Fig. 1D-F). The inverted cone technique was performed by removing additional cancellous bone laterally using the motion of the round burr at the dorsal level of the slot. Bone was firstly removed at the endplate of disc, then removal of bone progressed further cranially, reducing the cancellous bone until it was of eggshell thickness. Finally, the eggshell thickness dorsal cortical bone was lifted to gain access to the spinal cord.

\section{CT imaging}

Ten canine cervical vertebrae were imaged using CT unit (Alexion, TSX-034A; Toshiba Medical Systems, Japan) preoperatively and postoperatively, with $1.0 \mathrm{~mm}$ slice thickness, 0.8 slice interval, sharp reconstruction kernel, $\mathrm{kV} 120, \mathrm{~mA}$ 150. Scans were analyzed with DICOM viewing software (INFINITT; Infinitt Healthcare, Korea).

Measurements were taken from the transverse and sagittal slices. To measure the intact vertebral body length, measurements were taken from the preoperative sagittal plane in the point of the median sagittal remnant was defined as the length of the median vertebral body after the procedures (Figs. 2, 3).

Measurements of the transverse slice were taken from the widest window of the whole vertebral canal intact image at the cranial vertebral body (C3, C5). Vertebral canal width was defined as the greatest width of the vertebral canal at the measurement slice and the transverse window width was
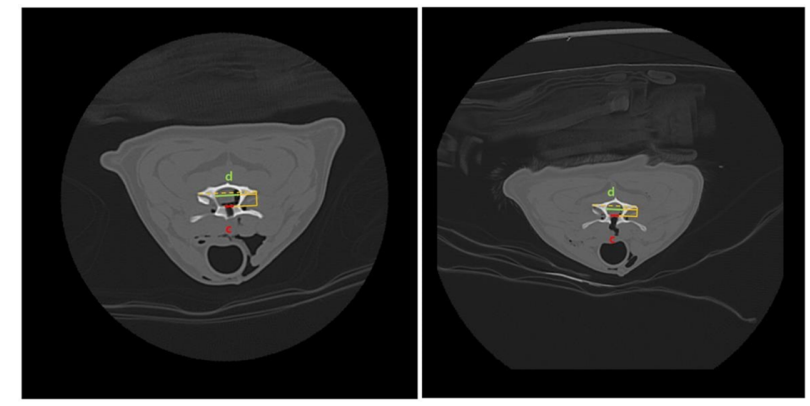

Fig. 4. Measurements taken from transverse slice in $\mathrm{C} 3$. (c) transverse window width, (d) vertebral canal width. Dashed line connects both side caudal articular process of C3, left (standard ventral slot), right (modified slanted ventral slot). C, cervical vertebra.

measured as defect width. All measurements were taken parallel to the line connecting the caudal articular process of the cranial vertebral body (Fig. 4).

Four variables (vertebral length, sagittal remnant, vertebral canal width, transverse window width) were measured at each surgery site. The total number of measurements was 100. All measurements were performed by a single reviewer. All measurements were linear and measured in $\mathrm{mm}$.

To control for variation in size, measurements were converted to percentages using vertebral length and sagittal remnant, vertebral floor width and transverse window width. The length to remnant ratio was defined as the ratio of the sagit- 
tal remnant to the vertebral length, and the transverse window width ratio was defined as the ratio of transverse window width to vertebral canal width.

\section{Statistical analyses}

To analyze the data for significant differences between the standard ventral slot group and the modified slanted ventral slot group, Statistical Package for the Social Sciences software with t-test was used. Statistical significance was set as $p<0.05$.

\section{Results}

Both the standard ventral slot and the modified slanted ventral slot procedures were able to expose the spinal cord successfully at the desired position. During most, but not all, of the standard ventral slot procedures, the window was directed at the median of the vertebral body.

\section{Preoperative data}

The estimated mean vertebral length was $21.8 \mathrm{~mm}$ for $\mathrm{C} 3$, $20.3 \mathrm{~mm}$ for $\mathrm{C} 4,18.3 \mathrm{~mm}$ for $\mathrm{C} 5$, and $16.6 \mathrm{~mm}$ for $\mathrm{C} 6$ (Table 1).

\section{Sagittal slice data}

The estimated length to remnant ratio (as estimated mean) in the standard ventral slot group was $86 \%$ (range, $83-88 \%$ )

Table 1. Value of intact vertebral length

\begin{tabular}{cc}
\hline \hline Cervical vertebra & Mean \pm SD $(\mathrm{mm})$ \\
\hline C3 & $21.8 \pm 2.0$ \\
C4 & $20.3 \pm 1.5$ \\
C5 & $18.3 \pm 1.7$ \\
C6 & $16.6 \pm 1.5$ \\
\hline
\end{tabular}

Data are shown as mean $\pm \mathrm{SD}$. in $\mathrm{C} 3,87 \%$ (range, $80-92 \%$ ) in $\mathrm{C} 4,81 \%$ (range, $79-83 \%$ ) in $\mathrm{C} 5$, and $84 \%$ (range, $82-89 \%$ ) in C6. The estimated length to remnant ratio (as estimated mean) in the modified slanted ventral slot group was $76 \%$ (range, $72-79 \%$ ) in $\mathrm{C} 3,99 \%$ (range, $99-100 \%$ ) in $\mathrm{C} 4,74 \%$ (range, $68-81 \%$ ) in $\mathrm{C} 5$, and $98 \%$ (range, 96-100\%) in C6 (Table 2). A statistically significant difference was observed in the length to remnant ratio between the standard ventral slot and the modified slated ventral slot techniques. The statistical difference in the ratio was significant in the standard ventral slot at 9.6\% $(p<0.05)$ in $\mathrm{C} 3$ and $7.4 \%(p<0.05)$ in $\mathrm{C} 5$. The difference in the ratio was significant in the modified slanted ventral slot at $11.8 \%$ $(p<0.05)$ in $\mathrm{C} 4$ and $14.6 \%(p<0.05)$ in C6.

\section{Transverse slice data}

The estimated transverse window width ratio (as estimated mean) in the standard ventral slot group was 34\% (range, 31$39 \%$ ) in $\mathrm{C} 3$ and 34\% (range, 30-38\%) in C5. The estimated transverse window width ratio (as estimated mean) in the modified slanted ventral slot group was 60\% (range, 53$69 \%$ ) in $\mathrm{C} 3$ and $58 \%$ (range, 55-62\%) in C5 (Table 2). A statistically significant difference was observed in the transverse window width ratio between the standard ventral slot and the modified slated ventral slot. The statistical difference in the ratio was significant in the standard ventral slot at $25.6 \%(p<0.05)$ in $\mathrm{C} 3$, and $24.2 \%(p<0.05)$ in C5 (Fig. 5).

Table 2. Value of length remnant ratio $(p<0.05)$

\begin{tabular}{ccc}
\hline \hline \multirow{2}{*}{ Cervical vertebra } & \multicolumn{2}{c}{ Length remnant ratio } \\
\cline { 2 - 3 } & Standard ventral slot & Slanted ventral slot \\
\hline C3 & $86 \pm 0.000$ & $76 \pm 0.005$ \\
C4 & $87 \pm 0.001$ & $99 \pm 0.034$ \\
C5 & $81 \pm 0.023$ & $74 \pm 0.000$ \\
C6 & $84 \pm 0.041$ & $98 \pm 0.018$ \\
\hline
\end{tabular}

Data are shown as mean \pm SD.

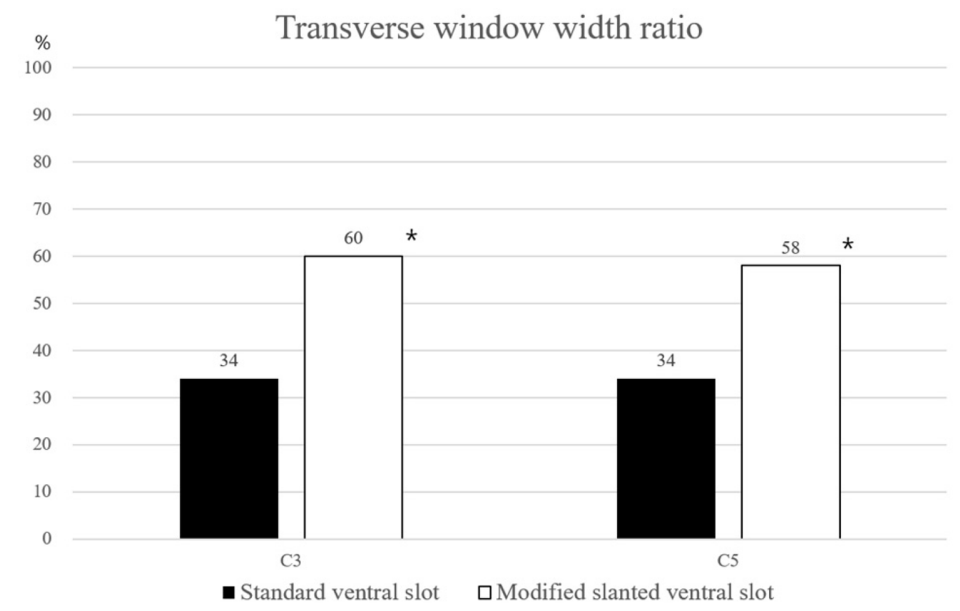

Fig. 5. Differences of measured transverse window width ratio. $\mathrm{C}$, cervical vertebra. *Asterisk indicates statistically different at $p<0.05$. 


\section{Discussion}

While one study show that modified slanted ventral slot had less complication [9], direct comparison of the access window between two methods was not studied. According to the results of this study, modified slanted ventral slot was more accessible to the cranial and lateral lesions of the cranial vertebral body as expected, but access to caudal vertebral lesion showed obvious limitation.

The differences in the Length remnant ratio between two procedures were different between cranial vertebral body and caudal vertebral body based on the surgery site. In the cranial vertebral body, $9.6 \%$ in $\mathrm{C} 3,7.4 \%$ in $\mathrm{C} 5$ was measured more longer in standard ventral slot than modified ventral slot and $11.8 \%$ in $\mathrm{C} 4,14.6 \%$ in C6 both maintaining almost intact vertebral body length was measured more longer in slanted ventral slot than standard ventral slot The clinical relevance of this is more accessible to the spinal cord in the cranial vertebral body by using slanted ventral slot and access to the caudal vertebra lesion is limited in slanted ventral slot because ostectomy is not performed on the caudal vertebra. Thus, disc material that persists caudal to the disc may be difficult to remove completely using the modified slanted slot procedure. To overcome these limitations, in 2007 McCartney [9] modified slanted ventral slot by performing fenestration disc and disc distraction. Applying periosteal elevator or small osteotome through the window in ventral annulus fibrosis with rotation assist remove of disc material.

Since ostectomy was not performed in the caudal vertebral body, transverse window width ration was measured in the cranial vertebral body. In transverse slice data, $25.6 \%$ in $\mathrm{C} 3$, $24.2 \%$ in C5 was measured more wide in modified slanted ventral slot with inverted cone technique. In 1991, Goring et al. [11] reported inverted cone technique to provide maximal spinal cord decompression, induce minimal morbidity. The conventional slanted slot may have narrower window than standard ventral slot. By applying with inverted cone techniques, the inverted cone shape of window allows access to lateralized disc material about more $25 \%$ of vertebral canal than standard ventral slot.

Based on these results, modified slanted ventral slot could more accessible some lesions than standard ventral slot by performing smaller ostectomy, although cranial lesions of caudal vertebra were limited. Smaller window procedure could reduce post-operative morbidity and complications. In human studies 2005, Thomé et al. [12] reported less extensive removal of vertebra associated with lower morbidity in lumbar spinal stenosis and also in veterinary, it is trend that performing less invasive procedures as pediculectomy in thoracolumbar disc herniation [13]. Although in 2016, Yang et al. [14] reported biomechanical response did not differ between standard ventral slot and slanted ventral slot. But in 2007, McCartney [9] reported lower rate of complications and similar recovery times by using modified slanted ventral slot than standard ventral slot, thus better prognosis can be expected in modified slanted ventral slot by reducing the size of the bone defect in the cervical vertebra.

In this study, CT was used to compare two methods of removing disc materials when cervical vertebral IVDD occurred. CT has the following benefits in vertebral morphology analysis compared to conventional radiography. In human medicine, CT is more accurate method for determination of pedicle screw position than conventional radiography [15] and considered the gold standard in a single center retrospective study of dogs with vertebral trauma [16]. In other study, CT significantly improves an evaluator's ability to identify vertebral canal penetration caused implantation [17]. Other benefits of CT evaluation of the vertebral canal include producing transverse images that can evaluate the vertebral canal in transverse slice and elimination of superimposed structures [18]. CT provides transverse images of vertebral canal anatomy with excellent bony detail, thus we evaluated vertebral canal by using CT images.

There are several limitations of this study. First, there may be variations of the cervical vertebra depending on breeds and size [19]. In this study, we selected beagle breed to minimizing bone variation. In clinical case, it may be necessary to apply the results of this study through careful preoperative diagnostic image. Second, because of cadaveric study, we could not consider the hemorrhage that can cause complications in the clinical case. Internal venous plexus is located on the ventral side of vertebral canal and diverge slightly from the ventral location at the intervertebral space levels [20]. Thus, excessive additional lateral cancellous bone removal may cause hemorrhage by insulting internal venous plexus in inverted cone technique. Third, only a single image reviewer performed the CT measurements. Although reviewers do not have a biased perspective, there may be a little error of measurement.

In conclusion, this study described limits of window access provided by standard ventral slot and modified slanted ventral slot in the cervical vertebral canal. According to the results, although smaller ostectomy was performed in the modified slanted ventral slot, some lesions were more accessible, but limitations were obvious in C3-4 and C5-6 intervertebral disk space. Thus, after the disc material has been identified through accurate preoperative diagnostic imaging, less morbidity and complications can be expected if the appropriate surgical method is selected based on the lesion of compression.

\section{References}

1. Bergknut N, Smolders LA, Grinwis GCM, Hagman R, Lagerstedt AS, Hazewinkel HAW, Tryfonidou MA, Meij BP. Intervertebral disc degeneration in the dog. Part 1: anatomy and physiology of the intervertebral disc and characteristics of intervertebral disc degeneration. Vet J 2013;195:282-291.

2. Coates JR. Intervertebral disk disease. Vet Clin North Am Small Anim Pract 2000;30:77-110.

3. Cherrone KL, Dewey CW, Coates JR, Bergman RL. A 
144 Ho Young Kang, Jinsu Kang, Haebeom Lee, Namsoo Kim, Suyoung Heo

retrospective comparison of cervical intervertebral disk disease in nonchondrodystrophic large dogs versus small dogs. J Am Anim Hosp Assoc 2004;40:316-320.

4. Johnston SA, Tobias KM. Veterinary Surgery: Small Animal. Elsevier, St. Louis, 2018.

5. Schmied O, Golini L, Steffen F. Effectiveness of cervical hemilaminectomy in canine Hansen type I and type II disc disease: a retrospective study. J Am Anim Hosp Assoc 2011; 47:342-350.

6. Boydell P, Boydell P. Horner's syndrome following cervical spinal surgery in the dog. J Small Anim Pract 1995;36:510512 .

7. Clark DM. An analysis of intraoperative and early postoperative mortality associated with cervical spinal decompressive surgery in the dog. J Am Anim Hosp Assoc 1986;22:739744 .

8. Lemarié RJ, Kerwin SC, Partington BP, Hosgood G. Vertebral subluxation following ventral cervical decompression in the dog. J Am Anim Hosp Assoc 2000;36:348-358.

9. McCartney W. Comparison of recovery times and complication rates between a modified slanted slot and the standard ventral slot for the treatment of cervical disc disease in 20 dogs. J Small Anim Pract 2007;48:498-501.

10. Bojrab MJ. Current Techniques in Small Animal Surgery. Teton New Media, Jackson, 2014.

11. Goring RL, Beale BS, Faulkner RF. The inverted cone decompression technique: a surgical treatment for cervical vertebral instability "wobbler syndrome" in Doberman pinschers. Part I. J Am Anim Hosp Assoc 1991;27:403-409.

12. Thomé C, Zevgaridis D, Leheta O, Bäzner H, PöcklerSchöniger C, Wöhrle J, Schmiedek P. Outcome after lessinvasive decompression of lumbar spinal stenosis: a randomized comparison of unilateral laminotomy, bilateral laminotomy, and laminectomy. J Neurosurg Spine 2005;3:129-141.

13. Werner T, McNicholas WT, Kim J, Baird DK, Breur GJ. Aplastic articular facets in a dog with intervertebral disk rupture of the 12th to 13th thoracic vertebral space. J Am Anim Hosp Assoc 2004;40:490-494.

14. Yang H, Lambrechts NE, Lehner M, Adam GM, Packer RA, Moore TW, Main RP. Effects of conventional and slanted ventral slot procedures on the biomechanical behavior of the C5-C6 vertebral motion unit in dogs. Am J Vet Res 2016;77: 846-853.

15. Learch TJ, Massie JB, Pathria MN, Ahlgren BA, Garfin SR. Assessment of pedicle screw placement utilizing conventional radiography and computed tomography: a proposed systematic approach to improve accuracy of interpretation. Spine 2004; 29:767-773.

16. Kinns J, Mai W, Seiler G, Zwingenberger A, Johnson V, Caceres A, Valdés-Martínez A, Schwarz T. Radiographic sensitivity and negative predictive value for acute canine spinal trauma. Vet Radiol Ultrasound 2006;47:563-570.

17. Hettlich BF, Fosgate GT, Levine JM, Young BD, Kerwin SC, Walker M, Griffin J, Maierl J. Accuracy of conventional radiography and computed tomography in predicting implant position in relation to the vertebral canal in dogs. Vet Surg 2010;39:680-687.

18. Sande RD. Radiography, myelography, computed tomography, and magnetic resonance imaging of the spine. Vet Clin North Am Small Anim Pract 1992;22:811-831.

19. Lincoln JD. Cervical vertebral malformation/malarticulation syndrome in large dogs. Vet Clin North Am Small Anim Pract 1992;22:923-935.

20. Gómez M, Freeman L, Jones J, Lanz O, Arnold P. Computed tomographic anatomy of the canine cervical vertebral venous system. Vet Radiol Ultrasound 2004;45:29-37. 